\title{
Use of Rostral Characters for Identifying Adult Billfishes (Teleostei: Perciformes: Istiophoridae and Xiphiidae)
}

\author{
Harry L. Fierstine and Nicole L. Voigt
}

\begin{abstract}
Seven species of the family Istiophoridae and Xiphias gladius were identified using only features of their rostrum. In the Istiophoridae, two rostral regions were emphasized, one-fourth and one-half the distance between the distal tip and the orbital margin of the lateral ethmoid bone. Characters studied in each region were the depth and width of rostrum and height, width, and position of nutrient canals (as seen in cross-section). Characters studied without reference to region were the distribution of denticles on both dorsal and ventral surfaces of the bill and position of the prenasal bone. In the Xiphiidae, the only characters studied were the depth and width of the rostrum at the level of the dermethmoid bone and the presence and placement of central chambers as seen in radiographs. $A$ total of $\mathbf{3 2}$ characters were analyzed as ratios using both multivariate and univariate statistics. The rostrum of $X$. gladius was separated from the Istiophoridae by its flat shape, Tetrapturus angustirostris from all other istiophorids by its widely separated nutrient canals, and the complex of $T$. audax/T. pfluegeri/ Makaira nigricans/M. indica from the complex of Istiophorus platypterus/T. albidus by having a smaller area of denticles on the dorsal surface. Tetrapturus pfluegeri was separated from $T$. audax, $M$. nigricans, and $M$. indica by having a longer denticle-free midline on the ventral surface of the rostrum. Tetrapturus audax was separated from $M$. nigricans and $M$. indica by the location of its nutrient canals. The complexes of Makaira nigricans/M. indica and $I$. platypterus/T. albidus were each separated using multivariate discriminant analysis. We show the study has application in identifying rostral fragments found as fossils and impaled in animate and inanimate objects such as marine turties and wooden ships and should have application wherever rostral fragments are found.
\end{abstract}

$T^{1}$ HE Istiophoridae and Xiphiidae are known collectively as billfishes because of the elongate rostrum or bill. Gross differences in rostral shape between the two families are well known, with the monotypic Xiphiidae having a flat, edentulous, blunt-tipped rostrum and the Istiophoridae a round, denticulated, pointed bill (Nakamura, 1983). Inter- and intrafamilial differences in rostral morphology have not been studied in detail even though neontologists and paleontologists sometimes identify billfish from rostral fragments (Fierstine, 1990; Frazier et al., 1994).

Bills from extant fishes are found in archaeology deposits (Poplin, 1975) and stomach contents (pers. com., P. Speare, Australian Institute of Marine Science, Townsville, Queensland, unpubl.) and impaled in animate and inanimate objects such as bales of rubber (Smith and Heemstra, 1986), a deep-diving vessel (Mather, 1976), boats and ships (Gudger, 1940; Chhapgar, 1972), fish (Voss, 1953; Goadby, 1975), 
marine turtles (Eckert et al., 1994; Frazier et al., 1994), whales (Major, 1981), and humans (Anonymous, 1966). Most billfish identifications seem to have been based on the following assumptions: if the rostrum were flat, it belonged to a swordfish; large and round, it belonged to either the black marlin (Makaira indica) or blue marlin (M. nigricans); and small and round, it belonged to a sailfish (Istiophorus platypterus).

Rostra are one of the more common fossil remains of the Istiophoridae and Xiphiidae and have been used to identify specimens to genus (Fierstine and Applegate, 1968) and species (Arambourg, 1927; Schultz, 1987; Woodward, 1901). Schultz (1987) even proposed a classification scheme of extant and extinct billfishes based primarily on rostral characters. However, because it has not been established that extant genera and species can be identified using rostral characters alone, fossil identifications and classification schemes exclusively based on rostral characters of fragmentary material are suspect.

The following is a statistical analysis of measurements and observations of external and internal structures of the rostrum of extant, adult fishes of the Istiophoridae and Xiphiidae. The study is designed to identify rostra, whether they are whole or incomplete segments.

\section{Materials and Methods}

Rostra were studied from the following species (the number of specimens examined are in parentheses): sailfish, Istiophorus platypterus (38), black marlin, Makaira indica (8), blue marlin, $M$. nigricans (42), longbill spearfish, Tetrapturus pfluegeri (2), shortbill spearfish, T. angustirostris (3), striped marlin, T. audax (14), white marlin, $T$. albidus (16) and swordfish, Xiphias gladius (40). We use the scientific and common names of Robins et al. (1991), institutional abbreviations of Leviton et al. (1985), and a combination of the osteological terminology of Gregory (1933), Gregory and Conrad (1937), Jollie (1986), Rojo (1991) and Schultz (1987) to maintain the more contemporary usage.

Istiophoridae.-Most rostra, other than those examined in museums, were collected at billfish tournaments by removing them at midorbit after taking whole body measurements. They were prepared by immersing them for a few minutes in a hot (approximately $90 \mathrm{C}$ ) solution of trisodium phosphate, then under warm, running tap water, carefully removing tissue with a stiff brush and forceps. This procedure was repeated until all tissue was removed. Next, the rostra were soaked overnight in $10 \%$ formalin solution (to inhibit spoilage) and dried for storage and study. Measurements were made with dial calipers and metric rule. Each rostrum was cut in cross-section at the $0.5 \mathrm{~L}$ and $0.25 \mathrm{~L}$ levels (see definitions below). Although most observations were made without magnification, low power magnification $(10 \mathrm{X})$ was used occasionally to determine the distribution of denticles and to obtain measurements of the nutrient canals.

No specimens of $T$. belone, $T$. georgei, $M$. indica larger than $164 \mathrm{~kg}$, and $M$. nigricans larger than $300 \mathrm{~kg}$ were available for examination. Skeletons were not studied if identifications, locality, or bill length data were suspect.

The study emphasizes two regions of the rostrum (Fig. 1): 0.5L or half the distance between the distal tip and the orbital margin of the lateral ethmoid bone, and $0.25 \mathrm{~L}$ or one-fourth the distance between the distal tip and the orbital margin of the lateral ethmoid bone. To avoid having to estimate $0.25 \mathrm{~L}$ and $0.5 \mathrm{~L}$, only complete rostra were examined. Characters studied (Figs. 1-2) in each region were depth (D) and width $(\mathrm{W})$ of rostrum, height $(\mathrm{H})$ and width $(\mathrm{N})$ of nutrient canal (as seen in cross-section), distance (DD) of the nutrient canal from the dorsal surface (as seen in cross-section), and distance (IC) between the nutrient canals (as seen in crosssection). Characters studied without reference to region were distribution of denticles on dorsal (DZ) and ventral (DVS) surfaces of the bill, position of the prenasal bone from the distal tip of the rostrum (P), presence or absence of denticles on the prenasal bone, and length from tip where fused premaxillae divide (VSPM) into separate bones.

In case there was asymmetry between the left and right nutrient canals and/or prenasals, only measurements of the left nutrient canal and prenasal were used in our calculations. If only one nutrient canal was present, any measurement involving the nutrient canal in that specimen was omitted from our analysis.

Xiphiidae.-Rostra were collected from commercial fishing vessels after each specimen had been transected anterior to the orbit. Bill length (tip of rostrum to orbital edge of the lateral ethmoid (prefrontal) bone) could not be measured. All specimens were frozen and later radiographed for presence and location of central chambers (Fig. 3). Depth (D) and width (W) of each rostrum were measured at the anterior extension of the dermethmoid bone (Fig. 3). 
(L)
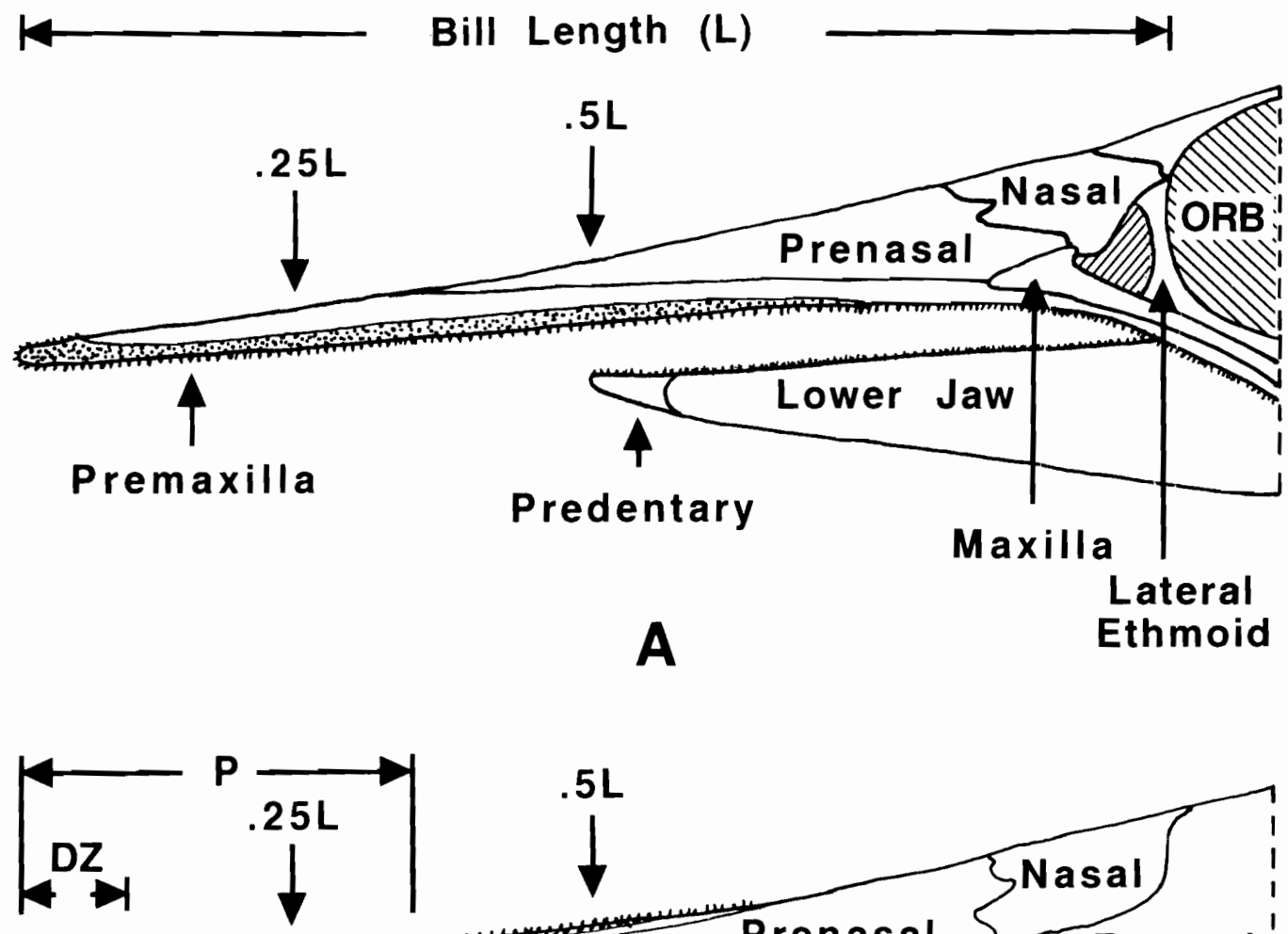

B
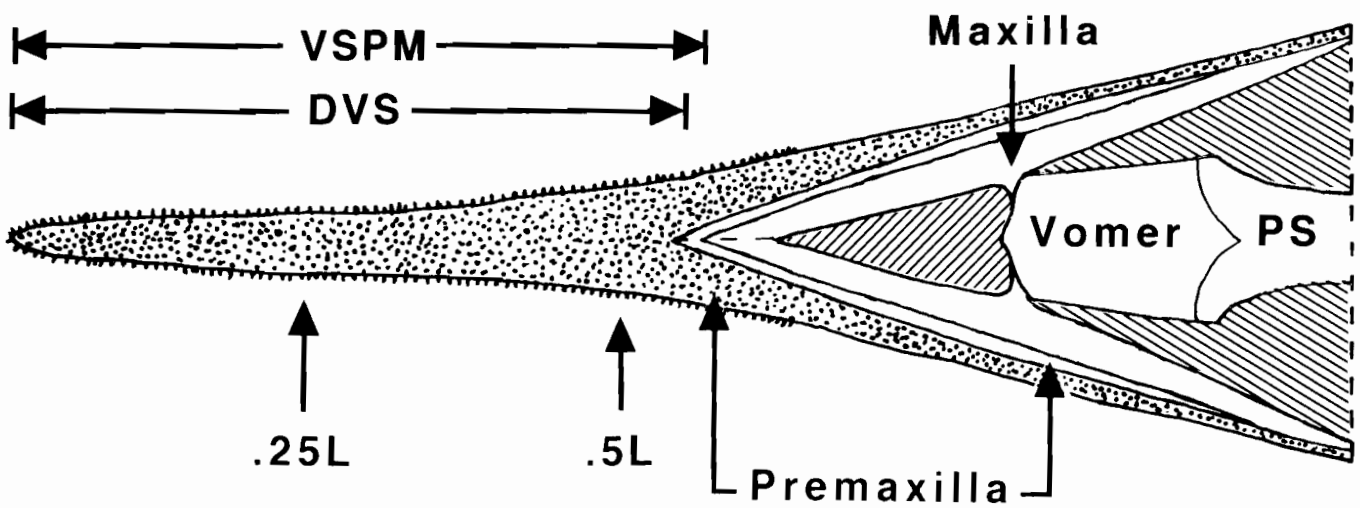

C 


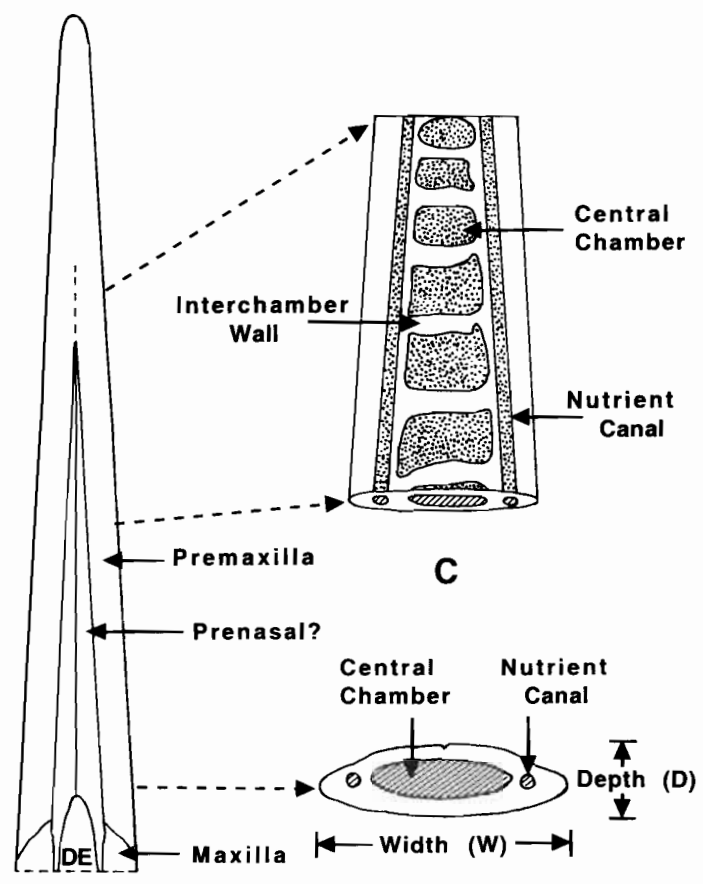

A

Fig. 2. Generalized rostrum of Xiphias gladius. (A) Dorsal view. (B) Enlarged cross-section from area just anterior (distal) to dermethmoid (DE) bone. (C) Enlarged longitudinal segment showing internal anatomy that might be visible in a radiograph.

Data analysis. - Multivariate analysis of variance (MANOVA) was used for initial analysis of the species. Resulting significant differences were then analyzed using univariate analysis of variance (ANOVA) with Tukey's pairwise multiple comparisons procedure. Because of missing data, ANOVA and MANOVA were applied using a general linear model procedure. Statistical Analysis Systems software (SAS Institute, Inc., 1988) was used for all analyses.

Because we believe ratios help reduce size effects and facilitate identification of isolated rostra or rostral fragments especially in keys, ratios were used as variables in all analyses. Atchley et al. (1976) stated that ratios do not remove size effects; however in their examples, each ratio always had the same variable in the denominator, whereas ours do not. However, the use of ratios often requires an arcsin of the square-root transformation because of the bi-

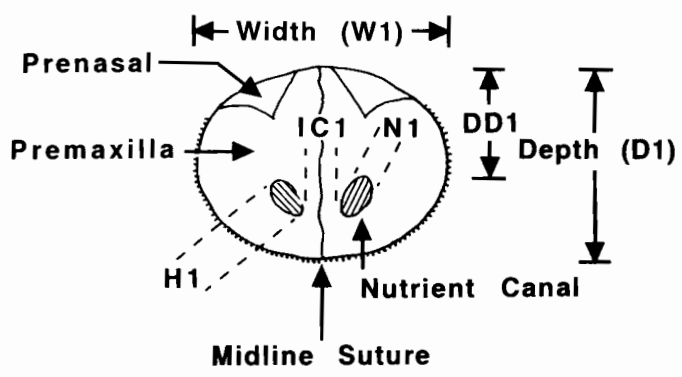

A

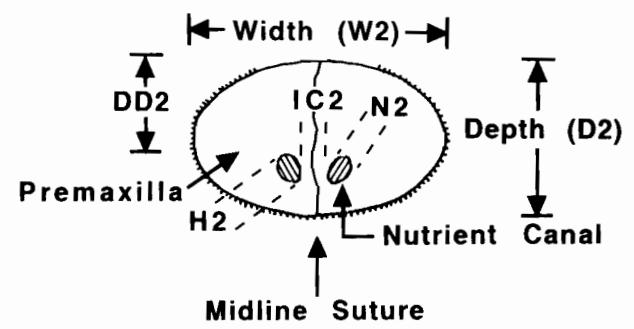

B

Fig. 3. Cross-section of generalized istiophorid rostrum at $(\mathrm{A})$ one-half bill length $(0.5 \mathrm{~L})$ and $(\mathrm{B})$ onefourth bill length $(0.25 \mathrm{~L})$. See text for definition of abbreviations.

nomial distribution of part to whole ratios (Sokal and Rohlf, 1981). Because the ratios used in this analysis do not represent a part divided by a whole, then they are not binomially distributed. Using the Shapiro-Wilks test, the majority of direct measurements and ratios were normally distributed. In those cases where there were substantial deviations from normality, the reported p-values should be considered approximations.

Tetrapturus angustirostris and T. pfluegeri were excluded from the species analysis because of small sample size, and $X$. gladius was removed from most statistical tests that analyzed variables other than depth (D) and width (W) of rostrum and presence or absence of central chambers. We omitted specimens from our statistical analyses if we did not section them (e.g., lacked permission).

Table 1, containing the observed ranges for

Fig. 1. Generalized istiophorid rostrum. (A) Lateral view. (B) Dorsal view. (C) Ventral view. DE $=$ Dermethmoid bone; $\mathrm{ORB}=$ Orbital region; $\mathrm{PS}=$ Parasphenoid bone. See text for definition of other abbreviations. 


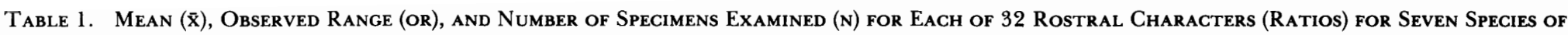
THE Family IstiophoridAe AND Xiphias gladius. Abbreviations for ratios are explained in text and Figures 1-3.

\begin{tabular}{|c|c|c|c|c|c|c|c|c|}
\hline Ratios & $\begin{array}{l}\text { 1. platypterus } \\
\overline{\bar{x}}(\mathrm{OR}) \mathrm{n}\end{array}$ & $\begin{array}{c}\text { M. indica } \\
\bar{x}(\mathrm{OR}) \mathrm{n}\end{array}$ & $\begin{array}{l}\text { M. nigricans } \\
\bar{x}(\mathrm{oR}) \mathrm{n}\end{array}$ & $\begin{array}{l}T_{\dot{\bar{x}}} \text { albidus } \\
\text { (oR } \mathbf{n}\end{array}$ & $\begin{array}{l}T \text {. angusti- } \\
\text { rostris } \\
\bar{x}(\mathrm{OR}) \mathrm{n}\end{array}$ & $\begin{array}{l}T \text {. audax } \\
\bar{x}(\mathrm{OR}) \mathrm{n}\end{array}$ & $\begin{array}{c}T . \text { pfluegeri } \\
\overline{\bar{x}}(\mathrm{OR}) \mathrm{n}\end{array}$ & $\begin{array}{c}X . \text { gladius } \\
\dot{\bar{x}}(\mathbf{O R}) \mathrm{n}\end{array}$ \\
\hline \multirow[t]{2}{*}{$\mathrm{D} 1 / \mathrm{W} 1$} & $\begin{array}{c}0.68 \\
(0.58-0.78)\end{array}$ & $\begin{array}{c}0.72 \\
(0.66-0.78)\end{array}$ & $\begin{array}{c}0.70 \\
(0.59-0.80)\end{array}$ & $\begin{array}{c}0.62 \\
(0.56-0.66)\end{array}$ & $\begin{array}{c}0.64 \\
(0.57-0.70)\end{array}$ & $\begin{array}{c}0.68 \\
(0.59-0.80)\end{array}$ & $\begin{array}{c}0.62 \\
(0.59-0.64)\end{array}$ & $\begin{array}{c}0.34 \\
(0.29-0.39)\end{array}$ \\
\hline & 31 & 8 & 41 & 13 & 3 & 13 & 2 & 40 \\
\hline \multirow[t]{2}{*}{$(\mathrm{D} 1 * \mathrm{~W} 1) .5 \mathrm{~L}$} & $\begin{array}{c}0.86 \\
(0.63-1.3)\end{array}$ & $\begin{array}{c}2.4 \\
(1.4-3.8)\end{array}$ & $\begin{array}{c}2.1 \\
(1.6-3.4)\end{array}$ & $\begin{array}{c}1.2 \\
(0.89-1.6)\end{array}$ & $\begin{array}{c}3.7 \\
(3.0-4.6)\end{array}$ & $\begin{array}{c}1.4 \\
(1.1-2.3)\end{array}$ & $\begin{array}{c}1.2 \\
(1.2-1.2)\end{array}$ & - \\
\hline & 31 & 8 & 41 & 13 & 3 & 13 & 2 & \\
\hline \multirow[t]{2}{*}{$\mathrm{D} 1 / .5 \mathrm{~L}$} & $\begin{array}{c}0.05 \\
(0.04-0.06)\end{array}$ & $\begin{array}{c}0.08 \\
(0.07-1.0)\end{array}$ & $\begin{array}{c}0.07 \\
(0.06-0.08)\end{array}$ & $\begin{array}{c}0.06 \\
(0.06-0.07)\end{array}$ & $\begin{array}{c}0.15 \\
(0.14-0.16)\end{array}$ & $\begin{array}{c}0.06 \\
(0.05-0.07)\end{array}$ & $\begin{array}{c}0.06 \\
(0.06-0.07)\end{array}$ & - \\
\hline & 31 & 8 & 41 & 13 & 3 & 13 & 2 & \\
\hline \multirow[t]{2}{*}{$\mathrm{W} 1 / .5 \mathrm{~L}$} & $\begin{array}{c}0.07 \\
(0.06-0.09)\end{array}$ & $\begin{array}{c}0.11 \\
(0.10-0.12)\end{array}$ & $\begin{array}{c}0.10 \\
(0.09-0.12)\end{array}$ & $\begin{array}{c}0.09 \\
(0.08-0.11)\end{array}$ & $\begin{array}{c}0.24 \\
(0.21-0.27)\end{array}$ & $\begin{array}{c}0.09 \\
(0.08-0.10)\end{array}$ & $\begin{array}{c}0.10 \\
(0.10-0.10)\end{array}$ & - \\
\hline & 31 & 8 & 37 & 13 & 3 & 13 & 2 & \\
\hline \multirow[t]{2}{*}{$\mathrm{H} 1 / \mathrm{D} 1$} & $\begin{array}{c}0.24 \\
(0.15-0.35)\end{array}$ & $\begin{array}{c}0.16 \\
(0.14-0.20)\end{array}$ & $\begin{array}{c}0.14 \\
(0.07-0.20)\end{array}$ & $\begin{array}{c}0.13 \\
(0.09-0.15)\end{array}$ & - & $\begin{array}{c}0.14 \\
(0.10-0.17)\end{array}$ & 一 & - \\
\hline & 28 & 7 & 36 & 7 & & 8 & & \\
\hline \multirow[t]{2}{*}{$\mathrm{N} 1 / \mathrm{W} 1$} & $\begin{array}{c}0.12 \\
(0.08-0.17)\end{array}$ & $\begin{array}{c}0.09 \\
(0.08-0.11)\end{array}$ & $\begin{array}{c}0.07 \\
(0.03-0.09)\end{array}$ & $\begin{array}{c}0.09 \\
(0.08-1.0)\end{array}$ & 一 & $\begin{array}{c}0.08 \\
(0.05-0.09)\end{array}$ & - & - \\
\hline & 28 & 7 & 35 & 7 & & 8 & & \\
\hline \multirow[t]{2}{*}{$(\mathrm{H} 1 * \mathrm{~N} 1) / .5 \mathrm{~L}$} & $\begin{array}{c}0.03 \\
(0.02-0.04)\end{array}$ & $\begin{array}{c}0.04 \\
(0.02-0.06)\end{array}$ & $\begin{array}{l}0.02 \\
(0-0.03)\end{array}$ & $\begin{array}{c}0.02 \\
(0.01-0.02)\end{array}$ & - & $\begin{array}{c}0.02 \\
(0.01-0.03)\end{array}$ & - & - \\
\hline & 28 & 7 & 35 & 7 & & 8 & & \\
\hline \multirow[t]{2}{*}{$\mathrm{DD} 1 / \mathrm{D} 1$} & $\begin{array}{c}0.32 \\
(0.21-0.41)\end{array}$ & $\begin{array}{c}\mathbf{0 . 4 3} \\
(0.38-0.49)\end{array}$ & $\begin{array}{c}0.46 \\
(0.33-0.62)\end{array}$ & $\begin{array}{c}0.40 \\
(0.35-0.45)\end{array}$ & - & $\begin{array}{c}0.11 \\
(0.08-0.14)\end{array}$ & - & - \\
\hline & 28 & 7 & 36 & 7 & & 7 & & \\
\hline \multirow[t]{2}{*}{$\mathrm{ICl} / \mathrm{W} 1$} & $\begin{array}{c}0.03 \\
(0.01-0.07)\end{array}$ & $\begin{array}{c}0.07 \\
(0.04-0.10)\end{array}$ & $\begin{array}{c}0.05 \\
(0.01-0.13)\end{array}$ & $\begin{array}{c}0.04 \\
(0.02-0.07)\end{array}$ & 一 & $\begin{array}{c}0.07 \\
(0.04-0.08)\end{array}$ & 一 & - \\
\hline & 26 & 7 & 29 & 7 & & 7 & & \\
\hline \multirow[t]{2}{*}{$(\mathrm{H} 1 * \mathrm{~N} 1) /(\mathrm{D} 1 * \mathrm{~W} 1)$} & $\begin{array}{c}9.6 \\
(4.1-18)\end{array}$ & $\begin{array}{c}12.8 \\
(5.2-24.0)\end{array}$ & $\begin{array}{c}3.7 \\
(0.60-7.4)\end{array}$ & $\begin{array}{c}4.9 \\
(3.7-5.9)\end{array}$ & - & $\begin{array}{c}6.9 \\
(3.3-13.0)\end{array}$ & - & - \\
\hline & 28 & 7 & 35 & 7 & & 8 & & \\
\hline \multirow[t]{2}{*}{$\mathrm{D} 2 / \mathrm{W} 2$} & $\begin{array}{c}0.64 \\
(0.55-0.75)\end{array}$ & $\begin{array}{c}0.68 \\
(0.59-0.77)\end{array}$ & $\begin{array}{c}0.64 \\
(0.54-0.83)\end{array}$ & $\begin{array}{c}0.53 \\
(0.47-0.61)\end{array}$ & $\begin{array}{c}0.61 \\
(0.58-0.64)\end{array}$ & $\begin{array}{c}0.61 \\
(0.52-0.67)\end{array}$ & $\begin{array}{c}0.65 \\
(0.65-0.65)\end{array}$ & - \\
\hline & 30 & 8 & 41 & 14 & 3 & 13 & 2 & \\
\hline
\end{tabular}


Table 1. Continued.

\begin{tabular}{|c|c|c|c|c|c|c|c|c|}
\hline Ratios & I. platypterus & $\begin{array}{l}\text { M. indica } \\
\bar{x}(\mathrm{oR}) \mathrm{n}\end{array}$ & $\begin{array}{l}\text { M. nigricans } \\
\bar{x}(\mathrm{OR}) \mathrm{n}\end{array}$ & $\begin{array}{l}T \cdot \text { albidus } \\
\dot{\bar{x}}(\text { OR }) \mathrm{n}\end{array}$ & $\begin{array}{l}\text { T. angusti- } \\
\text { rostris } \\
\bar{x}(\mathrm{OR}) \mathrm{n}\end{array}$ & $\begin{array}{l}\text { T. audax } \\
\dot{\vec{x}}(\mathrm{OR}) \mathrm{n}\end{array}$ & $\begin{array}{c}T \cdot \text { pfluegeri } \\
\bar{x}(\mathbf{O R}) \mathrm{n}\end{array}$ & $\begin{array}{l}X . \text { gladius } \\
\bar{x} \text { (OR) } n\end{array}$ \\
\hline \multirow[t]{2}{*}{$(\mathrm{D} 2 * \mathrm{~W} 2) / .25 \mathrm{~L}$} & $\begin{array}{c}0.98 \\
(0.74-1.4)\end{array}$ & $\begin{array}{c}5.3 \\
(3.1-7.8)\end{array}$ & $\begin{array}{c}0.83 \\
(0.57-1.2)\end{array}$ & $\begin{array}{c}\mathrm{I} .4 \\
(0.99-1.8)\end{array}$ & $\begin{array}{c}3.2 \\
(2.6-4.3)\end{array}$ & $\begin{array}{c}1.7 \\
(1.3-2.7)\end{array}$ & $\begin{array}{c}1.4 \\
(1.2-1.6)\end{array}$ & - \\
\hline & 30 & 8 & 36 & 14 & 3 & 13 & 2 & \\
\hline \multirow[t]{2}{*}{$\mathrm{D} 2 / .25 \mathrm{~L}$} & $\begin{array}{c}0.07 \\
(0.06-0.08)\end{array}$ & $\begin{array}{c}0.23 \\
(0.14-0.30)\end{array}$ & $\begin{array}{c}0.04 \\
(0.03-0.04)\end{array}$ & $\begin{array}{c}0.08 \\
(0.06-0.09)\end{array}$ & $\begin{array}{c}0.19 \\
(0.18-0.22)\end{array}$ & $\begin{array}{c}0.09 \\
(0.07-1.0)\end{array}$ & $\begin{array}{c}0.10 \\
(0.09-0.10)\end{array}$ & 一 \\
\hline & 31 & 8 & 36 & 14 & 3 & 13 & 2 & \\
\hline \multirow[t]{2}{*}{$\mathrm{W} 2 / .25 \mathrm{~L}$} & $\begin{array}{c}0.11 \\
(0.09-0.14)\end{array}$ & $\begin{array}{c}0.34 \\
(0.19-0.41)\end{array}$ & $\begin{array}{c}0.06 \\
(0.05-0.06)\end{array}$ & $\begin{array}{c}0.15 \\
(0.13-0.18)\end{array}$ & $\begin{array}{c}0.31 \\
(0.28-0.36)\end{array}$ & $\begin{array}{c}0.14 \\
(0.12-0.16)\end{array}$ & $\begin{array}{c}0.15 \\
(0.14-0.16)\end{array}$ & - \\
\hline & 30 & 8 & 36 & 14 & 3 & 13 & 2 & \\
\hline \multirow[t]{2}{*}{$\mathrm{H} 2 / \mathrm{D} 2$} & $\begin{array}{c}0.18 \\
(0.11-0.26)\end{array}$ & $\begin{array}{c}0.11 \\
(0.09-0.12)\end{array}$ & $\begin{array}{c}0.12 \\
(0.06-0.21)\end{array}$ & $\begin{array}{c}0.12 \\
(0.09-0.14)\end{array}$ & $\begin{array}{c}0.11 \\
(0.07-0.14)\end{array}$ & $\begin{array}{c}0.12 \\
(0.09-0.14)\end{array}$ & $\begin{array}{c}0.15 \\
(0.15)\end{array}$ & - \\
\hline & 28 & 7 & 32 & 7 & 2 & 8 & 1 & \\
\hline \multirow[t]{2}{*}{$\mathrm{N} 2 / \mathrm{D} 2$} & $\begin{array}{c}0.14 \\
(0.07-0.25)\end{array}$ & $\begin{array}{c}0.08 \\
(0.05-0.12)\end{array}$ & $\begin{array}{c}0.09 \\
(0.05-0.16)\end{array}$ & $\begin{array}{c}0.10 \\
(0.05-0.17)\end{array}$ & $\begin{array}{c}0.07 \\
(0.06-0.08)\end{array}$ & $\begin{array}{c}0.09 \\
(0.07-0.12)\end{array}$ & $\begin{array}{c}0.07 \\
(0.07)\end{array}$ & - \\
\hline & 28 & 7 & 32 & 7 & 2 & 8 & 1 & \\
\hline \multirow[t]{2}{*}{ N2/W2 } & $\begin{array}{c}0.09 \\
(0.05-0.17)\end{array}$ & $\begin{array}{c}0.06 \\
(0.03-0.08)\end{array}$ & $\begin{array}{c}0.06 \\
(0.04-0.10)\end{array}$ & $\begin{array}{c}0.05 \\
(0.03-0.09)\end{array}$ & $\begin{array}{c}0.04 \\
(0.03-0.05)\end{array}$ & $\begin{array}{c}0.06 \\
(0.04-0.07)\end{array}$ & $\begin{array}{c}0.05 \\
(0.05)\end{array}$ & - \\
\hline & 27 & 7 & 32 & 7 & 2 & 7 & 1 & \\
\hline \multirow[t]{2}{*}{$(\mathrm{H} 2 * \mathrm{~N} 2) / .25 \mathrm{~L}$} & $\begin{array}{c}0.02 \\
(0.01-0.03)\end{array}$ & $\begin{array}{c}0.04 \\
(0.01-0.06)\end{array}$ & $\begin{array}{l}0.01 \\
(0-0.01)\end{array}$ & $\begin{array}{c}0.01 \\
(0.01-0.02)\end{array}$ & $\begin{array}{l}0.01 \\
(0-0.02)\end{array}$ & $\begin{array}{c}0.01 \\
(0.01-0.02)\end{array}$ & $\begin{array}{c}0.01 \\
(0.01)\end{array}$ & - \\
\hline & 28 & 7 & 32 & 7 & 2 & 8 & 1 & \\
\hline \multirow[t]{2}{*}{$\mathrm{DD} 2 / \mathrm{D} 2$} & $\begin{array}{c}0.38 \\
(0.28-0.46)\end{array}$ & $\begin{array}{c}\mathbf{0 . 4 5} \\
(0.39-0.51)\end{array}$ & $\begin{array}{c}0.47 \\
(0.22-0.64)\end{array}$ & $\begin{array}{c}0.40 \\
(0.32-0.43)\end{array}$ & $\begin{array}{c}0.63 \\
(0.62-0.63)\end{array}$ & $\begin{array}{c}0.46 \\
(0.41-0.55)\end{array}$ & $\begin{array}{c}0.49 \\
(0.48-0.50)\end{array}$ & - \\
\hline & 28 & 7 & 32 & 7 & 2 & 8 & 2 & \\
\hline \multirow[t]{2}{*}{$\mathrm{IC} 2 / \mathrm{W} 2$} & $\begin{array}{c}0.14 \\
(0.60-0.21)\end{array}$ & $\begin{array}{c}0.13 \\
(0.11-0.16)\end{array}$ & $\begin{array}{c}0.12 \\
(0.04-0.25)\end{array}$ & $\begin{array}{c}0.16 \\
(0.13-0.20)\end{array}$ & $\begin{array}{c}0.43 \\
(0.40-0.45)\end{array}$ & $\begin{array}{c}0.18 \\
(0.13-0.25)\end{array}$ & $\begin{array}{c}0.24 \\
(0.24)\end{array}$ & - \\
\hline & 27 & 7 & 32 & 7 & 2 & 8 & 1 & \\
\hline \multirow[t]{2}{*}{$(\mathrm{H} 2 * \mathrm{~N} 2) /(\mathrm{D} 2 * \mathrm{~W} 2)$} & $\begin{array}{c}3.1 \\
(0.87-7.3)\end{array}$ & $\begin{array}{c}3.9 \\
(0.64-8.2)\end{array}$ & $\begin{array}{c}3.7 \\
(0.99-7.9)\end{array}$ & $\begin{array}{c}2.0 \\
(1.1-4.0)\end{array}$ & $\begin{array}{c}0.004 \\
(0.002-0.007)\end{array}$ & $\begin{array}{c}2.9 \\
(1.6-4.5)\end{array}$ & $\begin{array}{c}0.007 \\
(0.007)\end{array}$ & - \\
\hline & 27 & 7 & 32 & 7 & 2 & 8 & 1 & \\
\hline
\end{tabular}


TABle 1. Continued.

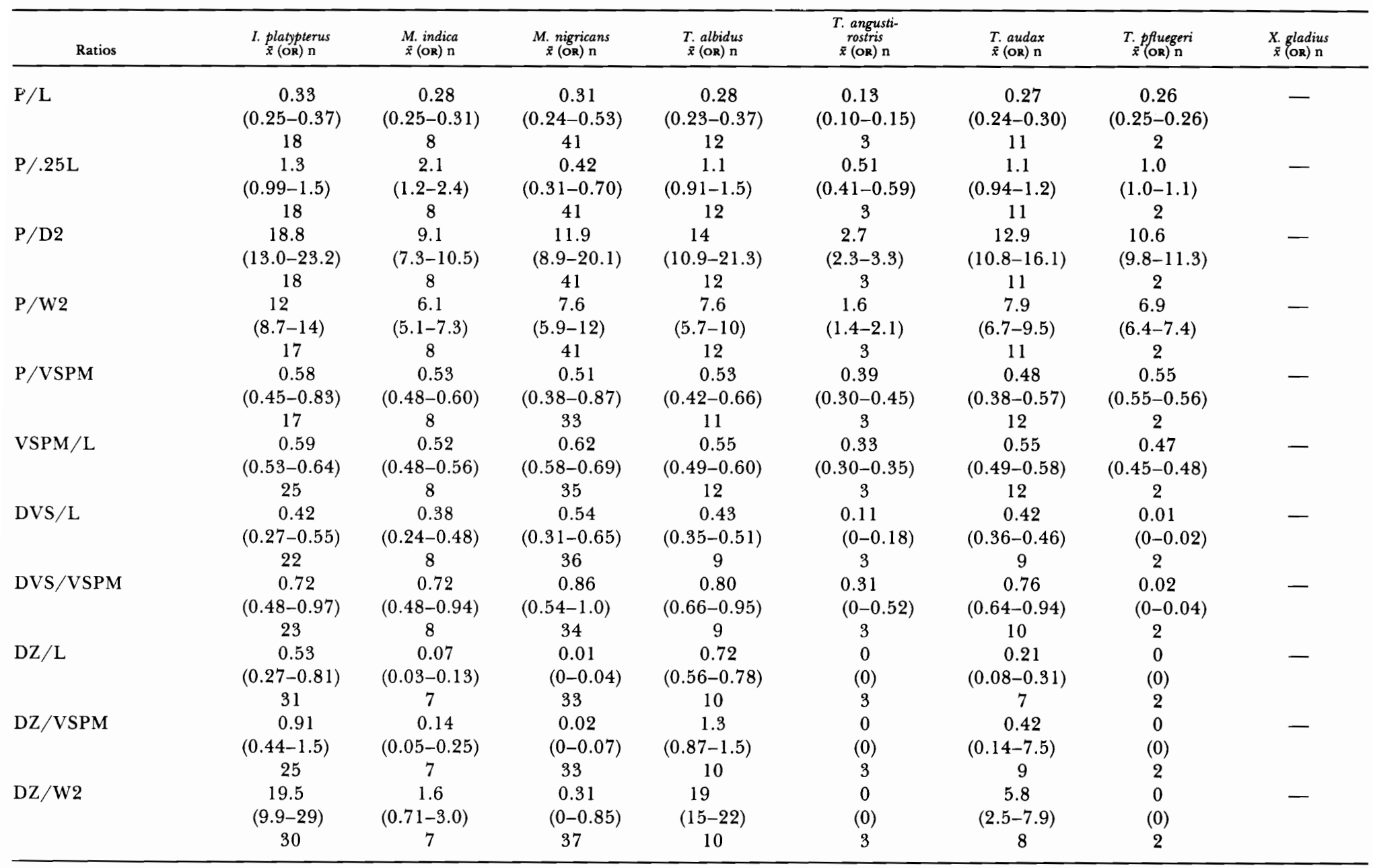


32 rostral characters (ratios) was the major source for constructing an identification key to eight species of billfishes. Species that could not be separated using these variables were separated by multivariate discriminant analysis. Error rates for the resulting functions were estimated using the resubstitituion method.

\section{Results}

Locating the position of $0.25 \mathrm{~L}$ or $0.5 \mathrm{~L}$ is a simple calculation in a whole rostrum (orbit to distal tip) but is more difficult to locate when the rostrum is incomplete. Knowing information about the prenasal bone and the ventral split in the premaxillae can help one decide whether the rostral segment contains either one of these regions or both. With the exception of the very short bill of $T$. angustirostris and a single specimen of $M$. nigricans, the distance from the tip of the bill to the distal extension of the prenasal bone is generally $0.23-0.40 \mathrm{~L}(\mathrm{P} / \mathrm{L}$ in 'Table 1 ). Since the prenasal bone tapers distally (Fig. 1B), it is noticeably large in cross-section at $0.5 \mathrm{~L}$ (Fig. 2A) and usually absent or tiny in cross-section at $0.25 \mathrm{~L}$ (Fig. $2 \mathrm{~B}$ ). The paired premaxillae converge toward the midline to form a sutured articulation (Figs. 1C, 2A) at or just proximal to $0.5 \mathrm{~L}$ (VSPM/L in Table 1 ).

Sometimes we observed asymmetry between the left and right nutrient canals and/or left and right prenasals in what otherwise appeared to be a normally developed rostrum. For example, in a specimen of $M$. nigricans (LACM 25411 ), the left nutrient canal at $0.25 \mathrm{~L}$ was over twice the height $(\mathrm{H})$ of the right canal, and the right prenasal was longer ( $\mathrm{P}$ was shorter) than the left. In a specimen of $T$. angustirostris (LACM 25499), the right nutrient canal was absent when sectioned at $0.25 \mathrm{~L}$. In a specimen of $M$. nigricans (LACM 25414), the left canal was situated dorsolateral to the right canal instead of lateral to it.

Tukey's pairwise multiple comparison procedure (Table 2 ) found many significant differences $(P<0.05)$ in the means of 32 rostral characters (ratios) that might prove useful in identifying species of the Istiophoridae. Istiophorus platypterus had the most ratios with means that were extremely significantly different $(P<$ $0.001)$ from other species, whereas the species comparison of $T$. albidus and $T$. audax had the smallest number of ratios with means that were extremely significantly different $(P<0.001)$.

An identification key was constructed primarily from variables with nonoverlapping ranges (Table 1). Variables with bill length components (e.g., L, $0.5 \mathrm{~L}$, and $0.25 \mathrm{~L}$ ) were not used in the key because, if one were attempting to identify a bill fragment, the length component would not be known. Xiphias gladius was separated from other billfishes by its edentulous, flat rostrum $(\mathrm{D} 1 / \mathrm{W} 1<0.5)$. Central chambers were usually present (Table 3; Fig. 3) along the entire length of the rostrum. Tetrapturus angustirostris differed from other species of the Istiophoridae by having a rostrum without denticles on its dorsal surface and with widely separated nutrient canals at $0.25 \mathrm{~L}$ (IC2/W2) (Table 1).

Two pairs of species (T. albidus vs I. platypterus and $M$. nigricans vs $M$. indica) could not be separated by variables with nonoverlapping ranges and were analyzed using 14 variables (ratios) in a stepwise discriminant analysis. The $M$. indica / $M$. nigricans analysis found four variables $[\mathrm{DZ}$ / $\mathrm{W} 2 ; \mathrm{P} / \mathrm{D} 2 ;(\mathrm{H} 1 * \mathrm{~N} 1) /(\mathrm{D} 1 * \mathrm{~W} 1) ; \mathrm{DD} 2 / \mathrm{D} 2]$ which, when used in a discriminant function, correctly separated the two species $100 \%$ of the time. Similarly, the I. platypterus / T. albidus analysis revealed three variables $[(\mathrm{H}] * \mathrm{~N} 1)$ / (D1*W 1$) ; \mathrm{P} / \mathrm{W} 2$; (H2*N2)/(D2*W2)] which correctly separated the two species $95 \%$ of the time.

Tukey's pairwise multiple comparison procedure (Table 2), a univariate analysis, found the variable $\mathrm{DZ} / \mathrm{W} 2$ to have no significance in separating $M$. nigricans from $M$. indica. However, the stepwise discriminant analysis, a multivariate procedure, found the variable important when placed in an equation with other variables. In addition, we used $\mathrm{DZ} / \mathrm{W} 2$ as a secondary character in the key to distinguish $M$. nigricans from $M$. indica, because only one specimen of $M$. indica and three specimens of $M$. nigricans in our samples had values of $\mathrm{DZ} / \mathrm{W} 2$ that fell in the overlapping region of 0.7 and 1 .

\section{Key to Six Species of Adult ISTIOPHORIDAE AND XIPHIAS GLADIUS Using Rostral Characters}

1A. Rostrum Hattened in cross-section, its depth (D) less than half of width (W). Denticles absent. Central chambers usually present Xiphias gladius (Family Xiphiidae)

1B. Rostrum oval in cross-section, its depth (D) more than half of width $(W)$. Denticles present. Central chambers never present 2 (Family Istiophoridae)

2A. Intercanal distance/bill width at $0.25 \mathrm{~L}$ (IC2/W2) greater than 0.4 Tetrapturus angustirostris

2B. Intercanal distance/bill width at $0.25 \mathrm{~L}$ (IC2/W2) less than 0.3

3A. Length of denticles on middorsal surface from tip/bill width at $0.25 \mathrm{~L}(\mathrm{DZ} / \mathrm{W} 2)$ less than 10 
Table 2. Results of Tukey's Multiple Comparison Procedure to Determine Significant Differences in the Means of 32 Rostral Characters (Ratios) among Five Species of the family Istiophoridae. Abbreviations for ratios are explained in text and in Figures 1-3. Abbreviations for species are as follows: Istiophorus platypterus $=\mathrm{Ip} ;$ Makaira indica $=\mathrm{Mi}$; . nigricans $=\mathrm{Mn} ;$ Tetrapturus albidus $=\mathrm{Tal} ;$ T. audax $=\mathrm{Tau}$. Abbreviations indicating significance are: ns $=$ not significant $(P>0.05) ; *=P<0.05 ; * *=P<0.01 ; * * *$

$$
=P<0.001 \text {. }
$$

\begin{tabular}{|c|c|c|c|c|c|c|c|c|c|c|}
\hline \multirow[b]{2}{*}{ Ratios } & \multicolumn{10}{|c|}{ Pairwise species comparisons } \\
\hline & Ip vs & $\begin{array}{l}\text { Ip vs } \\
\mathrm{Mn}\end{array}$ & $\begin{array}{l}\text { Ip vs } \\
\text { Tal }\end{array}$ & $\begin{array}{c}\text { Ip vs } \\
\text { Tau }\end{array}$ & $\begin{array}{c}\text { Mi vs } \\
\text { Mn }\end{array}$ & $\begin{array}{c}\text { Mi vs } \\
\text { Tal }\end{array}$ & $\begin{array}{l}\text { Mi vs } \\
\text { Tau }\end{array}$ & $\begin{array}{c}\text { Mn vs } \\
\text { Tal }\end{array}$ & $\begin{array}{c}\text { Mn vs } \\
\text { Tau }\end{array}$ & $\begin{array}{c}\text { Tal vs } \\
\text { Tau }\end{array}$ \\
\hline $\mathrm{D} 1 / \mathrm{W} 1$ & $* *$ & $*$ & ns & $*$ & ns & $* *$ & ns & $* *$ & ns & $* *$ \\
\hline$(\mathrm{D} 1 * \mathrm{~W} 1) / .5 \mathrm{~L}$ & $* * *$ & $* * *$ & ns & $*$ & ns & $* * *$ & $* *$ & $* * *$ & $* *$ & ns \\
\hline $\mathrm{D} 1 / .5 \mathrm{~L}$ & $* * *$ & $* * *$ & $* * *$ & $* * *$ & $* *$ & $* * *$ & $* * *$ & $* * *$ & $* * *$ & ns \\
\hline $\mathrm{W} 1 / .5 \mathrm{~L}$ & $* * *$ & $* * *$ & $* * *$ & $*$ & $* *$ & $* * *$ & $* * *$ & $*$ & $* * *$ & ns \\
\hline $\mathrm{Hl} / \mathrm{Dl}$ & $* * *$ & $* * *$ & $* * *$ & $* * *$ & ns & ns & ns & ns & ns & ns \\
\hline $\mathrm{N} 1 / \mathrm{W} 1$ & $* * *$ & $* * *$ & $* * *$ & $* * *$ & $*$ & ns & ns & * & ns & ns \\
\hline$(\mathrm{H} 1 * \mathrm{~N} 1) / .5 \mathrm{~L}$ & ns & $*$ & $* * *$ & $* *$ & $*$ & $* * *$ & $*$ & ns & ns & ns \\
\hline $\mathrm{DD} 1 / \mathrm{Dl}$ & $* * *$ & $* * *$ & $* * *$ & $* * *$ & $*$ & ns & $* * *$ & $*$ & $* * *$ & $* * *$ \\
\hline $\mathrm{IC} 1 / \mathrm{W} 1$ & $* * *$ & ns & ns & $* *$ & ns & $*$ & ns & ns & ns & $*$ \\
\hline$(\mathrm{H} 1 * \mathrm{~N} 1) /(\mathrm{D} 1 * \mathrm{~W} 1)$ & $* * *$ & $* * *$ & $* * *$ & $* * *$ & $* * *$ & ns & ns & ns & ns & ns \\
\hline $\mathrm{D} 2 / \mathrm{W} 2$ & ns & ns & ns & ns & ns & $* *$ & ns & $* *$ & ns & ns \\
\hline$(\mathrm{D} 2 * \mathrm{~W} 2) / .25 \mathrm{~L}$ & $* * *$ & ns & ns & ns & $* * *$ & $* * *$ & $* * *$ & ns & $*$ & ns \\
\hline $\mathrm{D} 2 / .25 \mathrm{~L}$ & $* * *$ & $* * *$ & $* *$ & $* *$ & $* * *$ & $* * *$ & $* * *$ & $* * *$ & $* * *$ & ns \\
\hline $\mathrm{W} 2 / .25 \mathrm{~L}$ & $* * *$ & $* * *$ & $* * *$ & $* *$ & $* * *$ & $* * *$ & $* * *$ & $* * *$ & $* * *$ & ns \\
\hline $\mathrm{H} 2 / \mathrm{D} 2$ & $* * *$ & $* * *$ & $* * *$ & $* * *$ & ns & ns & ns & ns & ns & ns \\
\hline $\mathrm{N} 2 / \mathrm{D} 2$ & $* * *$ & $* *$ & $* * *$ & $* * *$ & ns & ns & ns & ns & ns & ns \\
\hline $\mathrm{N} 2 / \mathrm{W} 2$ & $* *$ & $* *$ & $* *$ & $* *$ & ns & ns & ns & ns & ns & ns \\
\hline$(\mathrm{H} 2 * \mathrm{~N} 2) / .25 \mathrm{~L}$ & ns & $* *$ & $*$ & ns & $* * *$ & $* * *$ & $* * *$ & ns & ns & ns \\
\hline $\mathrm{DD} 2 / \mathrm{D} 2$ & $*$ & $* * *$ & ns & $* *$ & ns & ns & ns & $* *$ & ns & $*$ \\
\hline $\mathrm{IC} 2 / \mathrm{W} 2$ & ns & ns & $*$ & $* * *$ & ns & ns & $* *$ & ns & $* *$ & ns \\
\hline$(\mathrm{H} 2 * \mathrm{~N} 2) /(\mathrm{D} 2 * \mathrm{~W} 2)$ & $* * *$ & $* * *$ & $* * *$ & $* * *$ & ns & ns & ns & ns & ns & ns \\
\hline $\mathrm{P} / \mathrm{L}$ & $* *$ & ns & $*$ & $* *$ & $* *$ & ns & ns & * & $* *$ & ns \\
\hline $\mathrm{P} / .25 \mathrm{~L}$ & $* * *$ & $* * *$ & $* *$ & $* * *$ & $* * *$ & $* * *$ & $* * *$ & $* * *$ & $* * *$ & ns \\
\hline $\mathrm{P} / \mathrm{D} 2$ & $* * *$ & $* * *$ & $* * *$ & $* * *$ & $* *$ & $* * *$ & $* *$ & ns & ns & ns \\
\hline $\mathrm{P} / \mathrm{W} 2$ & $* * *$ & $* * *$ & $* * *$ & $* * *$ & $* *$ & $*$ & $*$ & ns & ns & ns \\
\hline P/VSPM & ns & ns & ns & $*$ & ns & ns & ns & ns & ns & ns \\
\hline VSPM /L & $* *$ & $* *$ & $* *$ & ns & $* * *$ & ns & ns & $* * *$ & $* * *$ & ns \\
\hline $\mathrm{VSP} / \mathrm{L}$ & ns & $* * *$ & ns & ns & $* * *$ & ns & * & $* * *$ & $* * *$ & ns \\
\hline DVS/VSPM & ns & $* * *$ & ns & ns & $* * *$ & * & * & $* *$ & * & ns \\
\hline $\mathrm{DZ} / \mathrm{L}$ & $* * *$ & $* * *$ & $* * *$ & $* * *$ & ns & $* * *$ & * & $* * *$ & $* * *$ & $* * *$ \\
\hline DZ/VSPM & $* * *$ & $* * *$ & $* * *$ & $* * *$ & ns & $* * *$ & * & $* * *$ & $* * *$ & $* * *$ \\
\hline $\mathrm{DZ} / \mathrm{W} 2$ & $* * *$ & $* * *$ & ns & $* * *$ & ns & $* * *$ & $*$ & $* * *$ & $* *$ & $* * *$ \\
\hline
\end{tabular}

3B. Length of denticles on middorsal surface from tip/bill width at $0.25 \mathrm{~L}(\mathrm{DZ} / \mathrm{W} 2)$ more than 10

4A. Length from tip of denticles on midventral surface/length of fused rostrum (DVS/ VSPM) less than 0.1 . Cross-sectional area of nutrient canal/cross-sectional area of bill at $0.25 \mathrm{~L}(\mathrm{H} 2 * \mathrm{~N} 2) /(\mathrm{D} 2 * \mathrm{~W} 2)$ less than 0.1 Tetrapturus pflueger

4B. Length from tip of denticles on midventral surface/length of fused rostrum (DVS/ VSPM) greater than 0.1. Cross-sectional area of nutrient canal/cross-sectional area of bill at $0.25 \mathrm{~L}(\mathrm{H} 2 * \mathrm{~N} 2) /(\mathrm{D} 2 * \mathrm{~W} 2)$ greater than 0.1 5

5A. Distance from the dorsal surface of the bill to the dorsal margin of the nutrient canal
(DD1)/bill depth (D1) at $0.5 \mathrm{~L}$ less than 0.2 Tetrapturus audax

7 5B. Distance from the dorsal surface of the bill to the dorsal margin of the nutrient canal (DD1)/bill depth (D1) at $0.5 \mathrm{~L}$ greater than 0.2 6

6A. Cross-sectional area of nutrient canal/crosssectional area of bill at $0.5 \mathrm{~L}(\mathrm{H} 1 * \mathrm{~N} 1)$ (D $\left.{ }^{*} \mathrm{~W} 1\right)$ less than 7.4. Length of denticles on middorsal surface from tip/bill width at $0.25 \mathrm{~L}(\mathrm{DZ} / \mathrm{W} 2)$ less than 1

Makaira nigricans

6B. Cross-sectional area of nutrient canal/crosssectional area of bill at $0.5 \mathrm{~L}(\mathrm{H} 1 * \mathrm{~N} 1)$ / $(\mathrm{D} 1 * \mathrm{~W} 1)$ greater than 5 . Length of denticles on middorsal surface from tip/bill width at $0.25 \mathrm{~L}(\mathrm{DZ} / \mathrm{W} 2)$ greater than 0.7 , usually 
Table 3. Frequency and Location of Central Chambers in the Rostrum of Xiphias gladius $(\mathrm{n}=33)$ Captured Off San Diego, California.

\begin{tabular}{lllccc}
\hline & \multicolumn{5}{c}{ Chamber configuration } \\
\cline { 2 - 6 } \multicolumn{1}{c}{ Sex } & None & $\begin{array}{c}1 / 4 \\
\text { Length }\end{array}$ & $\begin{array}{c}1 / 2 \\
\text { Length }\end{array}$ & $\begin{array}{c}\text { s/4 } \\
\text { Length }\end{array}$ & $\begin{array}{c}\text { Entire } \\
\text { length }\end{array}$ \\
\hline Male & 0 & 1 & 1 & 0 & 6 \\
Female & 1 & 5 & 4 & 0 & 15 \\
$\Sigma(\%$ of $n)$ & $1(3 \%)$ & $6(18 \%)$ & $5(15 \%)$ & 0 & $21(64 \%)$ \\
\hline
\end{tabular}

greater than 1 Makaira indica

6C. If cross-sectional area of nutrient canal/ cross-sectional area of bill at $0.5 \mathrm{~L}(\mathrm{H} 1 * \mathrm{~N} 1) /$ $\mathrm{D} 1 * \mathrm{~W} 1)$ is between 5.0 and 7.4 , and length of denticles on middorsal surface from tip/ bill width at $0.25 \mathrm{~L}(\mathrm{DZ} / \mathrm{W} 2)$ is 0.7 to 1 , then solve the following equations:

Equation $1=-153.31+4.59[\mathrm{DZ} / \mathrm{W} 2]+$ $8.26[\mathrm{P} / \mathrm{D} 2]+1490[(\mathrm{H} 1 * \mathrm{~N} 1) /(\mathrm{D} 1 * \mathrm{~W} 1)]$ $+392.57[\mathrm{DD} 2 / \mathrm{D} 2]$

Equation $2=-135.54+14.89[\mathrm{DZ} / \mathrm{W} 2]$ $+6.61[\mathrm{P} / \mathrm{D} 2]+1906[(\mathrm{Hl} * \mathrm{~N} 1) /(\mathrm{D} 1 * \mathrm{~W} 1)]$ $+358.60[\mathrm{DD} 2 / \mathrm{D} 2]$

If Equation $1>$ Equation 2, then Makaira nigricans

If Equation $2>$ Equation 1, then Makaira indica

7A. Nutrient canal height/bill depth at $0.5 \mathrm{~L}$ (H1/D1) always greater than 0.14 Istiophorus platypterus

7B. Nutrient canal height/bill depth at $0.5 \mathrm{~L}$ $(\mathrm{H} 1 / \mathrm{Dl})$ always less than 0.15 Tetrapturus albidus

7C. If nutrient canal height/bill depth at $0.5 \mathrm{~L}$ $(\mathrm{H} 1 / \mathrm{D} 1)$ is $0.14-0.15$, then solve the following equations:

Equation $1=-39.71+537.16[(\mathrm{H} 1 * \mathrm{~N} 1) /$

$(\mathrm{D} 1 * \mathrm{~W} 1)]+4.89[\mathrm{P} / \mathrm{W} 2]+378.10$ $[(\mathrm{H} 2 * \mathrm{~N} 2) /(\mathrm{D} 2 * \mathrm{~W} 2)]$

Equation $2=-13.38+214.56[(\mathrm{H} 1 * \mathrm{~N} 1) /$

$(\mathrm{D} 1 * \mathrm{~W} 1)]+3.03[\mathrm{P} / \mathrm{W} 2]+222.28$ [(H2*N2)/D2*W2)]

If Equation $1>$ Equation 2, then Istiophorus platypterus

If Equation $2>$ Equation 1, then Tetrapturus albidus

\section{Discussion}

This study demonstrates that extant species of the families Istiophoridae and Xiphiidae can be identified from whole or partial rostra. We envision the information to be useful in a variety of ways. For example, Frazier et al. (1994) described a case where a female olive ridley turtle,
Lepidochelys olivacea, was found with a fragment of rostrum protruding from its carapace. The fragment was identified as belonging to a sailfish, I. platypterus, because in cross-section the rostrum had relatively high nutrient canals as compared to the height of the rostrum. The reader had no quantitative information with which to evaluate the identification. If the exact measurements had been given $(\mathrm{Hl}=2.9 \mathrm{~mm}$, $\mathrm{D} 1=10 \mathrm{~mm}$ ), then the reader could have compared the ratio $(\mathrm{H} 1 / \mathrm{Dl}=0.29)$ with those given in Table 1 and observe that only $I$. platypterus has $\mathrm{H} 1 / \mathrm{D} 1>0.20$. In another example, one of us (HLF) is reexamining a case reported by Gudger (1940) where three rostra were found embedded in a ship's timber. One of the rostral fragments has ratios of $\mathrm{H} 1 / \mathrm{D} 1=0.09$ and $\mathrm{DD} 1 /$ $\mathrm{D} 1=0.55$. Using Table 1 , the first value overlaps slightly with values for $M$. nigricans and $T$. albidus, but the second value lies only within the observed range of $M$. nigricans. Thus, coupled with its large size, the rostrum is identified as belonging to $M$. nigricans.

There are some limitations to this effort. First, two species of spearfishes were excluded from examination because skeletal material was unavailable to us. Fortunately, these are the least common of the istiophorid billfishes and, therefore, the least likely to be encountered. Tetrapturus belone is restricted to the Mediterranean Sea and is most common around Italy; $T$. george $i$ is rare and restricted to the western Mediterranean Sea and adjacent Atlantic Ocean (Nakamura, 1985).

Second, the lack of large-sized M. indica and $M$. nigricans is a more serious omission since their rostra are morphologically similar, with many overlapping characters (Table 1). Makaira indica has a shorter bill than $M$. nigricans (Nakamura, 1985), and there are few significant differences $(P<0.001$ in Table 3$)$ between them that do not include bill length as part of the ratio. Perhaps if we had been able to examine near equal sample sizes and a greater size range, interspecific differences would have been more apparent. 
Third, the key itself has some limitations in sse. To move through the key effectively, the ostrum must be intact and undamaged from ip to $0.5 \mathrm{~L}$. If the tip of the bill is missing, then $1.25 \mathrm{~L}, 0.5 \mathrm{~L}, \mathrm{DZ}$, and $\mathrm{P}$ must be estimated from reconstructed tip. If the segment to be idenified has neither tip nor lateral ethmoid, then he location of $0.25 \mathrm{~L}$ and/or $0.5 \mathrm{~L}$ must be esimated from the size or absence of the prenasal one. Table 1 will probably prove more useful han the key when identifying rostral segments.

There is some confusion as to which bones orm the billfish rostrum (Fierstine, 1990). In he Istiophoridae, the rostrum is composed of he unpaired dermethmoid and vomer and vaired frontals, lateral ethmoids, maxillae, prenaxillae, prenasals, and nasals (Fig. 1). Schultz 1987) was the first to recognize the prenasal cones, although most workers consider them a eparate part of the premaxillae (Gregory and Jonrad, 1937; Nakamura, 1985; Davie, 1990). $\mathrm{Ne}$ believe the prenasal is a distinct, paired elment present in all istiophorids. According to ?ojo (1991) the term prenasal has been used or a bone in the sensory canal of Lepisosteus, ut we think the name is more appropriate for ise here.

Gregory and Conrad (1937) concluded that he distal three-fourths of the bill in the Xipiidae is primarily composed of premaxillae with laired nasals on the dorsal surface. The proxmal one-fourth was said to be formed from the lermethmoid, frontals, maxiliae, lateral ethnoids, and vomer. After dissecting a juvenile wordfish, Conrad (1937) concluded that the rue nasals are "minute but well-formed bony lement[s] lying dorsad to the narial openings," nd the bones that Gregory and Conrad (1937) alled nasals were actually parts of the premaxlae. Most subsequent workers (Nakamura, 983, 1985; Poplin et al., 1976), except Johnson 1986), have followed Gregory and Conrad 1937) and not Conrad (1937). Schultz (1987) ielieved that both nasal and prenasal bones are resent in the adult. 'Thus, the bone we labeled 'prenasal?"' (Fig. 3A) is often considered part of the premaxilla, and its true identity needs to re resolved. One of us (HLF) examined two arval specimens in the cleared and stained colection of the US National Museum and oberved a prenasal bone in the developing bill of - platypterus but not in $X$. gladius. Although this bservation suggests the absence of the prenasal one in Xiphiidae, we recommend caution in orming an opinion until more larval $X$. gladius re studied.

When examining rostra, whether fossil or reent, it is important to recognize an abnormal condition and not accord it taxonomic importance. Fishers have caught billfish with various lengths of their rostra missing (Gudger, 1940; Morrow, 1951; Wisner, 1958). In those istiophorid rostra we examined with tips missing, the paired nutrient canals were usually exposed at the broken surface, and in a specimen of $M$. nigricans (LACM 25405) where the break was in the middle of the bill, not only were the nutrient canals visible but the distal extensions of the prenasal bones were resorbed to expose prenasal grooves in the premaxillae. Wisner (1958) figured a specimen of $T$. audax with an upcurved bill, and one of us (HLF) has observed a specimen of $M$. indica and learned of a specimen of 1 . platypterus (pers. comm. G. Kelley, International Gamefish Association, Pompano Beach, FL, unpubl.), with bifurcated rostra. None of these specimens were skeletonized; thus the effect of their deformities on the bones are not known. To the best of our knowledge, we are the first to describe asymmetry in the size and placement of the nutrient canals in the istiophorid rostrum.

Schultz (1987) proposed a classification scheme of extant and extinct billfishes that was based primarily on rostral characters. Other than criticism for using small sample sizes and not taking into account intraspecific and interspecific variations (Fierstine, 1990), his effort has not been critically reviewed. Our study allows a more thorough examination of some of his conclusions for extant fishes.

Schultz (1987) divided the extant Istiophoridae into two families (Istiophoridae and Tetrapturidae) primarily because the tetrapturids have a short rostrum with denticles arranged in two bands on its ventral surface, separated by an edentulous zone, and istiophorids have the entire ventral surface of the rostrum covered with denticles. Based on our meager data for $T$. angustirostris, the ventral surface of the rostrum may be edentulous $(0.00)$ or may cover over $50 \%(0.52)$ of the distance between the distal tip and the ventral split in the premaxillae (see DVS/VSPM in Table 1). In fact, all species may have an edentulous zone, the length of which varies considerably (Table 1 ). We do not think this character is useful for defining families.

Schultz (1987) also resurrected Pseudohistiophorus De Buen, 1950, and placed it in the Tetrapturidae with $T$. angustirostris as the type species. He characterized the genus as having a short rostrum with two large nutrient canals. Using computerized tomography, he studied cross-sections of a single rostrum of $T$. angustirostris taken $45 \mathrm{~mm}, 70 \mathrm{~mm}$, and $80 \mathrm{~mm}$ from 
the distal tip. All of his sections seem to have been taken where the premaxillae are separated, posterior to the region we studied at $0.25 \mathrm{~L}$ where the premaxillae are fused. He may have interpreted the fossa for the anterior extension of the maxillae as nutrient canals, because we think small nutrient canals can be seen lateral to each fossa, particularly in the section cut at $45 \mathrm{~mm}$. We found T. angustirostris (Table 1) to have small, widely spaced nutrient canals that were not larger than other species relative to the depth of the rostrum (H2/D2). Thus, our study does not support the diagnostic feature that Schultz (1987) provides for the type species of Pseudohistiophorus that Nakamura (1983) properly referred to the synonym of Tetrapturus.

Finally, Schultz (1987) described a new genus and species, Thalattorhynchus austriacus, Middle Miocene, Austria, from a distal rostral fragment and placed it in the extinct Xiphiorhynchidae. The distinguishing feature was a single, off center, longitudinal canal that was observed by computerized tomography, otherwise the specimen was very similar to an istiophorid rostrum. The single canal may be an anomaly similar to the single canal observed here in a $T$. angustirostris (LACM 25499) or the incipient condition in a $M$. nigricans (LACM 25411). In addition, the Istiophoridae is known from the Middle Miocene to Recent, whereas the fossil record of the Xiphiorhynchidae (excluding T. austriacus) is unknown after the Middle Oligocene (Fierstine, 1990). Therefore, based on rostral morphology and geochronology, we doubt that the specimen belongs to a distinct genus or should be placed in the Xiphiorhynchidae. Most likely the specimen belongs to one of the extant genera of the Istiophoridae.

\section{MATERIAl. EXAMined}

Museum numbers, and locality, sex and size (Istiophoridae: lower jaw to fork length or weight; Xiphiidae: postorbit to fork length) are given for each specimen.

Istiophorus platypterus. -37 specimens. Islamorada, Florida: LACM 25302, M, $1646 \mathrm{~mm}$; LACM 25303, sex unknown, $1646 \mathrm{~mm}$; LACM 25305, M, 1511 mm; LACM 25306, F, 1506 mm; LACM 25307, F, 1651 mm; LACM 25308 M, $1447 \mathrm{~mm}$; LACM 25309, M, $1435 \mathrm{~mm}$; LACM 25310, M, $1511 \mathrm{~mm}$; LACM 25311, F, $1600 \mathrm{~mm}$; LACM 25312, F, $1626 \mathrm{~mm}$; LACM 25313, F, $1701 \mathrm{~mm}$; LACM 25314, M, 1651 mm; LACM 25315, M, 1455 mm; LACM 25317, F, $1765 \mathrm{~mm}$; LACM 25322, M, $1612 \mathrm{~mm}$;
LACM 25325, F, $1791 \mathrm{~mm}$. Destin, Florida: LACM 37998-1, sex and size unknown. Key West, Florida: LACM 25326, F, $47.6 \mathrm{~kg}$. Charleston Harbor, South Carolina: LACM 25486, M, $1590 \mathrm{~mm}$; LACM 25487, F, 1830 mm. Bimini, Bahamas: LACM 25318, F, 1600 $\mathrm{mm}$. Baja California del Sur, Mexico: LACM 25395, sex and size unknown. Mazatalan, Mexico: LACM 25425, M, 1910 mm; LACM 25426, M, $1770 \mathrm{~mm}$; LACM 25427, F, $1970 \mathrm{~mm}$; LACM 25432, M, 1780 mm; LACM 25434, M, $1780 \mathrm{~mm}$; LACM 25435, F, $1890 \mathrm{~mm}$; LACM 25436, F, 2060 mm; LACM 25439, M, 2000 mm. Stradbroke Id., Queensland, Australia: LACM 25485, sex and size unknown. Cape Bowling Green, Queensland, Australia: LACM 25443, M, 1815 mm; LACM 25446, sex unknown, 1365 mm; LACM 25447, M, 1915 mm; LAGM 25448, F, 2010 mm; LACM 25449, M, $2025 \mathrm{~mm}$.

Makaira indica._Eight specimens. Cabo San Lucas, Baja California: LACM 25470, F, 2591 mm. Cairns, Queensland, Australia: LACM 25509, M, $2195 \mathrm{~mm}$. Cape Bowling Green, Queensland, Australia: L.ACM 25444, M, 1375 mm; LACM 25445, M, 1325 mm. Port Stephens, New South Wales, Australia: LACM 25465, M, $2120 \mathrm{~mm}$; LACM 25466, M, 1960 mm; LACM 25467, F, 1790 mm; LACM 25468, $\mathrm{M}, 1910 \mathrm{~mm}$.

Makaira nigricans._42 specimens. Kailua-Kona, Hawaii: LACM 25403, F, 2680 mm; LACM 25404, F, $2438 \mathrm{~mm}$; LACM 25405, F, 2673 mm; LACM 25406, M, 2089 mm; LACM 25407 , M, $2127 \mathrm{~mm}$; LACM 25409, M, $2121 \mathrm{~mm}$; LACM 25410, M, 1956 mm; LACM 25411, M, 2349 mm; LACM 25412, M, 2108 mm; LACM 25413, M, 2057 mm; LACM 25414, M, 2413 mm; LACM 25415, M, 1924 mm; LACM 25416 , M, $2362 \mathrm{~mm}$; LACM 25417, F, $2121 \mathrm{~mm}$; LACM 25418, M, 2089 mm; LACM 25456, M, 2454 mm; LACM 25457, M, 83.9 kg; LACM 25458, F, 2516 mm; LACM 25459, M, 1902 mm; LACM 25460, M, 2010 mm; LACM 25463, M, $2223 \mathrm{~mm}$; LACM 25464, M, $2257 \mathrm{~mm}$; LACM 25473, M, 2019 mm; LACM 25474, M, 1919 mm; LACM 25475, M, 1960 mm; LACM 25476, F, 2667 mm; LACM 25477, M, 1938 mm; LACM 25480, M, 66.4 kg; LACM 25481, F, $119 \mathrm{~kg}$; LACM 25484, F, $2740 \mathrm{~mm}$; LACM 25488 , F, $295.9 \mathrm{~kg}$; LACM 25489, F, $278.9 \mathrm{~kg}$; LACM 25490, F, $57.2 \mathrm{~kg}$; LACM 25491, F, $1727 \mathrm{~mm}$; LACM 25492, M, $2219 \mathrm{~mm}$. Bimini, Bahamas: LACM 25316, F, $2217 \mathrm{~mm}$; LACM 25321, F, 2210 mm. Treasure Cay, Abaco, Bahamas: LACM 25329, F, 2332 mm; LACM 
25330, F, $2223 \mathrm{~mm}$; LACM 25331, F, 2471 mm. $36^{\circ} 07^{\prime} \mathrm{N} 73^{\circ} 25^{\prime} \mathrm{W}$ : USNM 196019, sex and size unknown. Atlantic Ocean: NCSM 91, sex unknown, $124.7 \mathrm{~kg}$; NCSM uncataloged, sex unknown, $181.4 \mathrm{~kg}$. Indian Ocean; LACM 46023-1, F, $2720 \mathrm{~mm}$.

Tetrapturus albidus. -16 specimens. Pensacola, Florida: LACM 25400, F, 1740 mm; LACM 25401, M, $1640 \mathrm{~mm}$; LACM 25402, M, 1585 mm; LACM 25502, F, 1705 mm; LACM 25503, F, 1740 mm; LACM 25504, F, 1643 mm; LACM 25505, M, $1530 \mathrm{~mm}$; LACM $25506 \mathrm{~F}, 1615$ $\mathrm{mm}$; LACM 25507, F, $1670 \mathrm{~mm}$. Beaufort, North Carolina: NCSM uncataloged, sex unknown, $26.8 \mathrm{~kg}$. Newport, Rhode Island: USNM 270769, sex and size unknown. Woods Hole, Massachusetts: USNM 270770, sex and size unknown. Western North Atlantic Ocean: USNM 270766, sex and size unknown; USNM 270767 , sex and size unknown; USNM 270768, sex and size unknown. Treasure Cay, Abaco, Bahamas: LACM 25332, F, $1765 \mathrm{~mm}$.

Tetrapturus angustirostris. - Three specimens. Kailua-Kona, Hawaii: LACM 25421, M, 1486 $\mathrm{mm}$; LACM 25422, sex and size unknown; LACM 25499, F, 1619 mm.

Tetrapturus audax.-14 specimens. San Diego, California: LACM 25493, F, 2124 mm; LACM 25494, F, 1924 mm; LACM 25495, F, 2006 mm; LACM 25496, F, 1990 mm; LACM 25497, F, $1943 \mathrm{~mm}$; LACM 25498, F, $2121 \mathrm{~mm}$. Southern California: LACM 25483, size and sex unknown; LACM uncatalogued, F, $2463 \mathrm{~mm}$; LACM uncatalogued, sex unknown, $2352 \mathrm{~mm}$; LACM uncatalogued, F, Size unknown. $8^{\circ} 49^{\prime}$ S 833'ㄹ: USNM 270773, sex unknown, 1990 mm. Bermagui, New South Wales, Australia: LACM 25501, F, $2500 \mathrm{~mm}$. Bay of Islands, New Zealand: LACM 25500, M, $2420 \mathrm{~mm}$. Poor Knight's Island, New Zealand: LACM 25508, F, $2650 \mathrm{~mm}$.

Tetrapturus pfluegeri.-Two specimens. Off Venezuela: LACM 25461, F, 1690 mm; LACM 25462, M, $1740 \mathrm{~mm}$.

Xiphias gladius. -40 specimens. San Diego, California: LACM 25349, F, $1640 \mathrm{~mm}$; LACM 25350, M, 1830 mm; LACM 25351, F, 1800 mm; LACM 25355, F, 1500 mm; LACM 25356 , F, $1640 \mathrm{~mm}$; LACM 25357, F?, $1380 \mathrm{~mm}$; LACM 25358, F, $1630 \mathrm{~mm}$; LACM 25359, F?, $1650 \mathrm{~mm}$; LACM 25360, F, $1540 \mathrm{~mm}$; LACM 25365, M?, $1480 \mathrm{~mm}$; LACM 25366, F, 1810 mm; LACM 25367, F, 1550 mm; LACM 25368,
F, 1890 mm; LACM 25369, F, 1900 mm; LACM $25370, \mathrm{~F}, 1830 \mathrm{~mm}$; LACM 25371, M, 1470 mm; LACM 25372, F, 2260 mm; LACM 25373 , F, $1920 \mathrm{~mm}$; LACM 25374, M, $1450 \mathrm{~mm}$; LACM 25375, F, $1850 \mathrm{~mm}$; LACM 25376, F, $1900 \mathrm{~mm}$; LACM 25377, M, $1850 \mathrm{~mm}$; LACM 25378, F, $1850 \mathrm{~mm}$; LACM 25379, F, 1550 mm; LACM 25380, F, 1310 mm; LACM 25381, F, 1940 mm; LACM 25382, F, 1840 mm; LACM 25383, F, $1920 \mathrm{~mm}$; LACM 25384, F, 1550 $\mathrm{mm}$; LACM 25385, M, 1450 mm; LACM 25386, M, $1640 \mathrm{~mm}$; LACM 25387, F, $1760 \mathrm{~mm}$; LACM 25388, M, 1660 mm; LACM 25389, F?, $1700 \mathrm{~mm}$; LACM 25390, M, $1720 \mathrm{~mm}$; LACM 25391, M?, $1850 \mathrm{~mm}$; LACM 25392, F, 2060 mm; LACM 25393, M, 1830 mm; LACM 25394, F, $1500 \mathrm{~mm}$; LACM 25482, F, $2050 \mathrm{~mm}$.

\section{ACKNOWLEDGMENTS}

This study could not have been completed without the assistance of others, especially $R$. Smidt who guided us with our statistical analysis and B. Collette, G. D. Johnson, and R. Rosenblatt who greatly improved the manuscript with their criticisms. R. Pimentel reviewed our use of ratios. G. D. Johnson brought the cleared and stained specimens to our attention and helped us interpret our observations.

F. Arocha, R. Brill, J. Contillo, D. Cupka, P. Davie, C. Daxboeck, M. Farber, G. Greenwald, M. Judge, R. McKay, J. Pepperell, R. Pitman, E. Prince, P. Pristas, R. Rosenblatt, P. Speare, J. Squire, S. Tibbo, and A. Van der Heiden helped obtain billfish for skeletal preparations. E. Prince (Southeast Fisheries Center, NMFS) deserves special recognition for help acquiring the elusive longbill spearfish and other Western Atlantic specimens. P. Fithian (Pacific Ocean Research Foundation) fostered cooperation between the Kona, Hawaii, fishing community and visiting scientists and provided facilities for preparing skeletons during several Hawaiian International Billfish Tournaments. R. Miranda (Bimini Big Game Club) was a gracious host during the 1994 Bacardi Rum Billfish Tournament. Special thanks to the following individuals for allowing examination of specimens in their custody: W. Anderson Jr., G. Burgess, D. Cantania, B. Collette, O. Crimmen, R. Feeney, L. Paimer, J. Paxton, and V. Schneider.

\section{Literature Cited}

Anonymous. 1966. Fish's sword removed from seadiver's skull. Med. World News (Sept. 30):38-39. Arambourg, C. 1927. Les Poissons fossiles d'Oran. 
Materiaux pour la Carte géologiques de l'Algerie. 1st Série, Paleéntologie. 6:1-298.

Archley, W. R., C. T. Gaskins, and D. Anderson. 1976. Statistical properties of ratios. I. Empirical results. Syst. Zool. 25:137-148.

ChHAPGar, B. F. 1972. A case of unprovoked attack by a fish on a boat. J. Bombay Nat. Hist. Soc. 69: 661-663.

Conran, G. M. 1937. The nasal bone and sword of the swordfish (Xiphias gladius). Am. Mus. Novitates 968:1-3.

DaviE, P, 1990. Pacific marlins: anatomy and physiology. Massey Univ, Press, Palmerston North, New Zealand.

Eckert, S., D. McDonald, and P. Dutton. 1994. Dermochelys coriacea (Leatherback Sea Turtle). Billfish interaction. Herpetological Rev. 25:63-64.

Fierstine, H. L. 1990. A paleontological review of three billfish families (Istiophoridae, Xiphiidae, and Xiphiorhynchidae), p. 11-19. In: Planning the future of billfishes. Part 2, Contributed papers. R. H. Stroud (ed.). Proc. Second International Billfish Symposium, Kailua-Kona, Hawaii, 1-5 Aug. 1988, National Coalition for Marine Conservation, Inc., Savannah, GA.

- AND S. P. APPLEGATE. 1968. Billfish remains from southern California with remarks on the importance of the predentary bone. B. Southern Calif. Acad. Sci. 67:29-39.

Frazier, J. G., H. L. Fierstine, S. C. Beavers, F. Aghaval, H. Suganuma, R. L. Pitman, Y. YaMaguchi, ANd C. M. Prigioni. 1994. Impalement of marine turtles (Reptilia, Chelonia: Cheloniidae and Dermochelyidae) by billfishes (Osteichthyes, Perciformes: Istiophoridae and Xiphiidae). Env. Biol. Fishes 39:85-96.

GoAdBy, P. 1975. Big fish and blue water: gamefishing in the Pacific. Angus and Robertson, Sydney, Australia.

Gregory, W. K. 1933. Fish skulls, a study of the evolution of natural mechanisms. Trans. Amer. Phil. Soc. 23:i-vii+75-481.

- AND G. M. Conrad. 1937. The comparative osteology of the swordfish (Xiphias) and the sailfish (Istiophorus). Amer. Mus. Novitates 952:1-25.

Gudger, E. W. 1940. The alleged pugnacity of the swordfish and the spearfishes as shown by their attacks of vessels. Mem. Royal Asiat. Soc. Bengal. 12: 215-315.

Johnson, G. D. 1986. Scombroid phylogeny: an alternative hypothesis. Bull. Mar. Sci. 39:1-41.

Jollie, M. 1986. A primer of bone names for the understanding of the actinopterygian head and pectoral girdle skeletons. Can. J. Zool. 64:365-379.

Leviton, A. E., R. H. Gibbs JR., E. Heal, and G. D. DAwson. 1985. Standards in herpetology and ichthyology: Part 1. Standard symbolic codes for institutional resource collections in herpetology and ichthyology. Copeia 1985:802-832.

Major, P. F. 1981. Combat on the high seas. Sea Frontiers 27:280-286.
Mather, C. O. 1976. Billfish. Saltaire Publ. Co., Sidney, Canada.

Morrow, J. E. 1951. A striped marlin (Makaira mitsukurii) without a spear. Copeia 1951:303-304.

Nakamura, I. 1983. Systematics of billfishes (Xiphiidae and Istiophoridae). Publ. Seto Mar. Biol. Lab. 28:255-396.

- 1985. An annotated and illustrated catalogue of marlins, sailfishes, spearfishes and swordfishes known to date. FAO Fisheries Synopsis no. 125. $5: i-i v+1-65$.

Poplin, C. , F. Poplin, And A. Ricqles. 1976. Quelques particularités anatomiques et histologiques du rostre de l'Espadon (Xiphias gladius L.). C. R. Seances Acad. Sci. Ser. D. 282:1105-1108.

Poplin, F. 1975. Restes de rostre d'Espadon trouves dans un gisement néolithique de l'etang de Leucate (Aude). Bull. Soc. Prehist. Fr. 72:69-70.

Robins, C. R., R. M. BAILEY, C. E. Bond, J. R. BrooKER, E. A. I AaCHNER, R. N. LEA, AND W. B. SCOTt. 1991. Common and scientific names of fishes from the United States and Canada. Amer. Fish. Soc. Spec. Pub. 20:1-183.

Rojo, A. L. 1991. Dictionary of evolutionary fish osteology. CRC Press, Boca Raton, FL.

SAS InstituTe, Inc. 1988. SAS/STAT user's guide. Release 6.03 ed. Statistical Analysis Systems Institute, Inc., Cary, NC.

Schultz, O. 1987. Taxonomische Neugruppierung der Überfamilie Xiphioidea (Pisces, Osteichthyes). Ann. Naturhist. Mus. Wien. Serie A. für Mineralogie und Petrographie, Geologie und Paläontologie, Anthropologie und Prähistories. 89:95-202.

Smith, M. M., and P. C. Heemstra (eds.). 1986. Smith's sea fishes. Macmillan South Africa Publishers Pty. Ltd., Johannesburg.

SokAl, R. R., AND F. J. Rohlf. 1981. Biometry. 2d ed. W. H. Freeman and Co., New York.

Voss, G. L. 1953. A contribution to the life history and biology of the sailfish, Istiophorus americanus Cuv. and Val., in Florida waters. Bull. Mar. Sci. Gulf and Caribbean 3:206-240.

WISNER, R. L. 1958. Is the spear of istiophorid fishes used in feeding? Pac. Sci. 12:60-70.

WoODWARD, A.S. 1901. Catalogue of the fossil fishes in the British Museum (Natural History). Vol. 4. British Museum (Natural History), London.

Biological Sciences Department, California Polytechnic State University, San Luis Obispo, California 93407. Present ADDRESS: (NLV) DEPARTMENT OF ENVIronmental, Population, and Organismic Biology, University of Colorado, Bollder, Colorado 80309-0334. Send reprint requests to HLF. Submitted: $25 \mathrm{Jan}$. 1995. Accepted: 12 May 1995. Section editor: R. Winterbottom. 